\title{
Transcodificación de numerales verbales a formato arábigo en educación básica primaria
}

Transcoding Verbal Numerals to Arabic Format in Primary Education

Transcodification de chiffres verbaux au format arabique dans l'éducation basique primaire

Transcodificação de numerais verbais ao formato arábico em educação básica primária

Fecha de recepción: 27 DE JUNIO DE 2014/Fecha de aceptación: 24 DE JULIO DE 2015/Fecha de disponibilidad en línea: 15 DE MARZO DE 2016

Encuentre este artículo en http://magisinvestigacioneducacion.javeriana.edu.co/

Escrito por Christian Hederich-Martínez Universidad Pedagógica Nacional Bogotá, Colombia hederich@pedagogica.edu.co

Ángela Camargo-Uribe Universidad Pedagógica Nacional Bogotá, Colombia acamargo@pedagogica.edu.co

\section{Resumen}

Angélica Avalo-Azcárate Universidad Pedagógica Nacional Bogotá, Colombia

El artículo reporta los resultados de una investigación que angelicaavalo@gmail.com examina el desarrollo de la tarea de transcodificación de numerales verbales hablados en castellano a formato arábigo. La evidencia proviene de una prueba de dictado de números aplicada a 118 estudiantes de los grados $1^{\circ}$ a $4^{\circ}$ de educación básica primaria. Los resultados indican que la eficacia conductual de los niños frente a la tarea sigue un proceso discontinuo marcado por ascensos y descensos bruscos a medida que se van incorporando numerales cada vez mayores. Se discuten algunas características de los numerales asociadas con la dificultad para su transcodificación.

\section{Palabras clave}

Desarrollo infantil; aprendizaje; procesamiento de la información; matemáticas; análisis numérico

\section{Transferencia a la práctica}

La comprensión de la naturaleza de la tarea de transcodificación de numerales, o "tarea de dictado de números", es fundamental para la interpretación de los errores cometidos por los niños al ejecutarla. De igual forma, la comprensión de esta tarea permitirá al docente el diseño de evaluaciones más ajustadas con las particularidades y características de los niños durante las fases de desarrollo en que esta tarea aparece como objetivo de la educación matemática. Los resultados muestran la lógica detrás de la mayoría de los errores cometidos, lo cual representa el punto inicial para su superación.

Para citar este artículo / To cite this article / Pour citer cet article / Para citar este artigo

Hederich-Martínez, C.; Camargo-Uribe, A. \& Ávalo-Azcárate, A. (2016). Transcodificación de numerales verbales a formato arábigo en educación básica primaria. magis, Revista Internacional de Investigación en Educación, 8 (17), 27-46. http://dx.doi.org/10.11144/Javeriana.m8-17.tnvf 


\section{Keywords}

Child development; learning; information processing; mathematics; numerical analysis

\section{Mots clés}

Développement enfantin; apprentissage; traitement de l'information; mathématiques; analyse numérique

\section{Abstract}

The article presents the results of a study that examines the task of transcoding spanish verbal numerals to Arabic numerals. The evidence stems from a number dictation test applied to 118 students from grades 1 to 4 . Results indicate that the behavioral effectiveness of children during the task follows a discontinuous process characterized by abrupt ascents and descents when increasing numerals are being incorporated. This paper discusses some characteristics of the numbers that are associated with the difficult task of being transcoded.

\section{Transfer to practice}

Understanding the nature of the task of transcoding numerals, or "task dictation of numbers" is key to interpret children mistakes. Similarly, understanding this task may allow teachers to design evaluations that best fit to the particularities and characteristics of children during their phases of development in which this task appears to be an objective within mathematics education. Results show the logic behind most of the mistakes, which is the starting point for its improvement
28

\section{Résumé}

L'article reporte les résultats d'une recherche qui examine le développement du devoir de transcodification de chiffres verbaux parlés en espagnol à format arabique. L'évidence provienne d'une preuve de dictée de chiffres appliquée à 118 étudiants de $1^{\circ}$ et $4^{\circ}$ d'éducation basique primaire Les résultats indiquent que l'efficacité de conduite des enfants en face du devoir suit un processus discontinu marqué par montées et descentes brusques au fur et à mesure qu'on ajoute de chiffres à chaque fois plus grandes: On discute les caractéristiques de chiffres associées avec la difficulté de transcodification

\section{Transfert à la pratique}

La compréhension de la nature du devoir de transcodification de chiffres, ou « devoir de dictée de numéros » est fondamentale pour l'interprétation de fautes faites pour les enfants lorsqu'ils le font. Ainsi, la compréhension de ce devoir permet à l'enseignant le dessein d'évaluations plus ajustés avec les particularités et caractéristiques des enfants pendant les moments du développement dans lequel ce devoir apparait en tant qu'objectif de l'éducation mathématique. Les résultats montrent la logique derrière la pluparts de fautes faites, cela représente le point initial pour les résoudre.

\section{Palavras-chave}

Desenvolvimento infantil; aprendizagem; processamento da informação; matemáticas; análise numérica

\section{Resumo}

O artigo apresenta os resultados de uma pesquisa que examina o desenvolvimento da tarefa de transcodificação de numerais verbais falados em castelhano ao formato arábico. A evidência foi reunida a partir de um ditado de números usado com 118 estudantes dos graus $1^{\circ}$ a $4^{\circ}$ de educação básica primária. Os resultados indicam que a eficácia das ações das crianças frente à tarefa tem um processo descontínuo marcado por momentos positivos e negativos bruscos à medida que se vão incorporando numerais cada vez maiores. Discutem-se algumas características dos numerais associadas com a dificuldade para sua transcodificação.

\section{Transferência à prática}

O entendimento da natureza da tarefa de transcodificação de numerais, ou "tarefa de ditado de números", é fundamental para a interpretação dos erros cometidos pelas crianças ao executá-la. Da mesma maneira, o entendimento desta tarefa permitirá ao docente o desenho de avaliações mais ajustadas às particularidades e características das crianças durante as fases de desenvolvimento em que esta tarefa aparece como objetivo da educação matemática. Os resultados mostram a lógica por trás da maioria dos erros cometidos, o que representa o ponto inicial para sua superação. 


\section{Introducción}

Cuando un maestro dice en voz alta un número, por ejemplo, "trescientos cuarenta y cinco" y su alumno lo escribe en formato arábigo: "345", este último está transcodificando la información numérica, es decir, está cambiando el código en el que esta información fue emitida — una expresión numérica verbal- por otro código de naturaleza semiográfica —un numeral arábigo—, que mantiene diferencias lingüísticas fundamentales con el primero. De igual forma, cuando leemos en voz alta un número escrito en formato arábigo también estamos transcodificando, pero en este caso, el código final es el verbal. Estudios realizados durante la década de 1980 encontraron que a los escolares les resulta más sencilla la tarea de lectura de símbolos arábigos, que su tarea de escritura a partir de la palabra dicha (Dickson, Brown \& Gibson, 1984).

Hay un acuerdo total en el sentido de que el proceso de transcodificación de numerales es absolutamente fundamental para los aprendizajes matemáticos posteriores (Moura, Wood, Pinheiro-Chagas, Lonnemann, Krinzinger, Willmes \& Haase, 2013; Zuber, Pixner, Moeller \& Nuerk, 2009), incluyendo el cálculo mental (Dehaene \& Cohen, 1995) y la comparación de magnitudes (Nuerk, Wenger \& Willmes, 2005).

En el presente artículo, examinaremos en detalle el desarrollo de la tarea de transcodificación de numerales de formato verbal hablado a formato arábigo, conocida en el argot escolar como "tarea de dictado de números", en grupos de niños de diferente grado escolar. En general, intentaremos apoyar la hipótesis de que el desarrollo de la transcodificación sigue un proceso discontinuo marcado por rupturas, ascensos y descensos bruscos en la eficacia conductual de los niños frente a la tarea, a medida que se van incorporando numerales cada vez mayores y se van construyendo marcos sintácticos más generales. Cada vez que el niño avanza un escalón más en su desarrollo hacia el manejo global del sistema de numeración, los marcos sintácticos utilizados para el rango numérico previo resultan inadecuados e/o insuficientes, por lo que podría presentarse un descenso en su eficacia conductual, indicado por el aumento de la frecuencia de errores en la escritura de números, incluso del rango anterior ya aprendido (Otálora \& Orozco, 2006).

Esta hipótesis es consistente con la descripción del proceso de desarrollo cognitivo hecha por la teoría de la redescripción representacional (Karmiloff-Smith, 1994). Desde el punto de vista de esta teoría, el niño hace sucesivas redescripciones representacionales del sistema que está aprendiendo, en versiones cada vez más flexibles y explícitas.

Otro de los alcances del artículo es el examen de las características particulares de los números que predicen sus niveles de complejidad. Este examen permite inferir la forma en que se van modificando los marcos sintácticos presentes para incorporar nuevas características que den cuenta de números de rangos mayores.

En un trabajo previo, que constituye el antecedente inmediato del presente estudio, se examinaron en detalle las características de los errores de transcodificación de numerales verbales a formato arábigo en que incurren niños escolarizados (Orozco \& Hederich, 1998). Esto permitió llegar a la propuesta de un conjunto de categorías ordenadas para la tipificación de las diferentes clases de errores, descritas por medio de los procesos cognitivos implicados en estos.

Como es usual en esta línea de trabajos, en esa ocasión partimos de la distinción hecha por Michael McCloskey (1992) entre errores léxicos
Descripción del artículo | Article description | Description de l'article | Artigo descrição

Este artículo reporta algunos resultados obtenidos en el proyecto Desarrollo de la transcodificación de numerales verbales a formato arábigo, cofinanciado por la Universidad Pedagógica Nacional en Bogotá (Colombia) y Colciencias. Código: 1108-1110359. En este artículo de investigación, los autores examinan cómo se va modificando la eficacia de los niños frente a las tareas de dictado de números y las discontinuidades que se dan durante el proceso, para relacionarlas con características particulares de los numerales dictados. 
y errores sintácticos y subcategorizamos los errores sintácticos en nueve tipos diferentes (Orozco \& Hederich, 2002).

Los errores léxicos hacen referencia a producciones que difieren de la versión correcta en uno o varios dígitos específicos, sin que se afecte la estructura posicional del número completo. Por ejemplo, "trescientos setenta y cinco" es transcodificado erróneamente como 365. Por su parte, en errores sintácticos, si bien la información léxica puede ser la acertada, la producción arábiga resultante difiere de la correcta en la longitud del numeral. Este es el caso, por ejemplo, en que el numeral "trescientos setenta y cinco" es transcodificado a formato arábigo como 300705, pues se observa una falla en el procesamiento sintáctico del numeral que denominamos, en su momento, como error de "yuxtaposición"1 (Orozco \& Hederich, 2002).

La distinción entre los errores léxicos y los sintácticos resulta particularmente importante para el estudio del proceso de desarrollo y/o aprendizaje de la transcodificación. Desde el punto de vista cognitivo, los errores léxicos se producen por una falla en la recepción, almacenamiento o recuperación de la información léxica referida a los dígitos, más que a sus posiciones. En determinadas circunstancias, este tipo de error puede ser cometido por sujetos expertos en la tarea, por lo que no resulta de mayor interés desde el punto de vista evolutivo. Por el contrario, los errores sintácticos revelan claramente un estado incipiente en la construcción del sistema de numeración decimal. En la medida en que estamos interesados en describir el proceso de aprendizaje de este sistema de numeración y de los códigos verbal y arábigo, nos concentraremos en los errores sintácticos cometidos por niños escolarizados sin requerimientos de atención especial.

Antes de presentar los resultados, debe exponerse con mayor profundidad la tarea de la transcodificación de numerales de formato verbal hablado a formato arábigo, lo cual se hará en el siguiente apartado. Hecho esto, pueden describirse los momentos típicos del aprendizaje escolar de los sistemas de numeración verbal y arábigo, y las dificultades que surgen en cada momento. Completada esta parte, podrán ser expuestos los elementos metodológicos y la evidencia empírica que sustenta la hipótesis.

\section{Transcodificar palabras numéricas}

La tarea de transcodificar consiste en pasar de expresiones morfosintácticas orales a expresiones numéricas escritas caracterizadas por la posición del numeral en potencias de diez. En términos psicolingüísticos, se trata de traducir de un sistema de reglas de marcación morfosintáctica (sufijos derivativos y morfemas composicionales de la lengua) a un sistema de reglas que utiliza el recurso de la posición para significar potencias de diez.

La transcodificación de expresiones numéricas verbales ha sido ampliamente analizada, en especial desde una óptica neuropsicológica, mediante el estudio de pacientes adultos que presentan lesiones cerebrales localizadas (Campbell \& Clark, 1988, 1992; Cipolotti \& Butterworth, 1995; Cohen \& Dehaene, 1995; Deloche \& Seron, 1987; McCloskey, 1992; Noël \& Seron, 1992).

Los resultados de estos trabajos se han expresado en la formulación de diferentes modelos teóricos del sistema de procesamiento numérico. Todos estos modelos han sido planteados desde el punto de vista de la modularidad de la mente, que postula la existencia de módulos de proce-

1 La yuxtaposición se define como el caso en que la expresión arábiga resulta de la correspondencia directa y completa con la expresión verbal hablada. 
samiento ubicados localmente que son específicos de dominio. En general, los diferentes modelos coinciden en los siguientes puntos: 1) los módulos de comprensión de numerales verbales y de numerales arábigos son diferentes y se encuentran funcionalmente separados; 2) igual ocurre con los módulos de producción de numerales verbales y arábigos; y 3) aunque no todos los autores lo admiten (McCloskey, Aliminosa \& Sokol, 1991), hay conexiones directas entre los módulos de comprensión de números verbales y arábigos y los de su producción, que no requieren como paso intermedio la comprensión completa del numeral expresado, sino de una estrategia algorítmica para recuperar de manera directa de la memoria de trabajo la forma del numeral arábigo (Barrouillet, Camos, Perruchet \& Seron, 2004). A este último proceso se le conoce como "transcodificación asemántica", y podemos suponer que representa el grado más alto de maestría en lo que a la tarea de transcodificación se refiere (Deloche \& Seron, 1982a, 1982b).

Un análisis detallado de la gramática del sistema de numeración verbal excede los alcances del presente artículo. Por lo pronto, baste notar que dentro de la expresión verbal deben diferenciarse dos tipos de partículas: las raíces, correspondientes a los dígitos, y los sufijos, que expresan sus posiciones. Obsérvese, por ejemplo, la estructura del numeral verbal "trescientos cuarenta y cinco":

Tabla 1

Comparación entre una expresión numérica verbal y su correspondiente numeral arábigo

\begin{tabular}{l|c}
\hline Expresión verbal & Trescientos cuarenta y cinco \\
\hline $\begin{array}{l}\text { Reemplazando las raíces, siempre } \\
\text { referidas a dígitos, por las cantidades } \\
\text { que denotan en sus símbolos arábigos: }\end{array}$ & 3-cientos 4-enta y 5 \\
\hline $\begin{array}{l}\text { Reemplazando los sufijos por operadores } \\
\text { multiplicativos de potencias de diez (el } \\
\text { sufijo "-cientos" indica una multiplicación } \\
\text { por 100 y el sufijo "-enta" indica una } \\
\text { multiplicación por 10) }\end{array}$ & $3(\times 100) 4(\times 10)$ y 5 \\
\hline $\begin{array}{l}\text { Las composiciones por yuxtaposición y } \\
\text { conjunción indican operaciones aditivas: }\end{array}$ & $3(x 100)+4(x 10)+5$ \\
\hline Expresión arábiga & 345 \\
\hline \hline
\end{tabular}

Fuente: elaboración propia

\section{El desarrollo de la transcodificación}

Cuando el niño llega a la escuela, tiene un manejo del sistema de numeración verbal —esto es, del sistema de palabras que se refieren a números - bastante desarrollado. El aprendizaje se centra entonces en el sistema numérico decimal y en las rutinas cognitivas y motrices que permiten su escritura. Normalmente, el proceso se da por rangos, o número de dígitos: se inicia por numerales de un dígito y aumenta progresivamente el número de dígitos del numeral en formato arábigo. El propósito es llegar a altos niveles de mecanización del proceso, en la idea de que este es el punto de partida para aprendizajes de más alto orden.

La evidencia obtenida en investigaciones realizadas con sujetos sin dificultades matemáticas específicas, y en particular con niños durante su proceso de aprendizaje, ha sido más bien reducida. De acuerdo con lo que 
se sabe sobre la tarea, la transcodificación de numerales es una competencia que presenta alguna dificultad y que toma un tiempo más o menos prolongado para su perfeccionamiento (Moura, Wood, Pinheiro-Chagas, Lonnemann, Krinzinger, Willmes \& Haase, 2013; Orozco, Guerrero \& Otálora, 2007; Orozco \& Hederich, 2002; Otálora \& Orozco, 2006). Algunos datos indican que se relaciona estrechamente con la capacidad de la memoria de trabajo (Barrouillet, Camos, Perruchet \& Seron, 2004; Camos, 2008; Moura, Wood, Pinheiro-Chagas, Lonnemann, Krinzinger, Willmes \& Haase, 2013) y con las particularidades de la lengua de la que se esté haciendo la transcodificación (Zuber, Pixner, Moeller \& Nuerk, 2009).

Siguiendo a William Haas (1996), el aprendizaje del sistema de numeración arábigo es más parecido al aprendizaje de una lengua extranjera, que a la adquisición de un dialecto escrito de la propia lengua materna; mientras, desde el punto de vista del niño, se trata de la construcción de estructuras sintácticas totalmente nuevas.

Lograr la escritura de números arábigos de una cifra supone básicamente dos aprendizajes: 1) la instauración de rutinas motrices específicas para cada signo numérico y 2) la identificación de la notación numérica como formalmente diferente de la notación alfabética, en lo que toca al carácter semiográfico de la primera. El niño debe aprender que, cuando se trata de números, cada símbolo posee un significado. Esta diferenciación formal se logra a muy temprana edad: de cuatro a cinco años (Tolchinsky, 1993). La distinción temprana entre la notación de números y letras se extiende incluso a rasgos sintácticos, lo cual se indica por el hecho de que un niño acepta como válida una secuencia de una cifra repetida, por ejemplo, "444", pero no acepta como válida una secuencia de una letra repetida, por ejemplo, "ttt" (Tolchinsky \& Karmiloff-Smith, 1993).

Al pasar al rango de números de dos cifras, las particularidades sintácticas del código arábigo comienzan a operar. En este momento puede hablarse de un inicio en el aprendizaje de las reglas generativas del sistema, pues cuando se trabaja con números de dos cifras se requiere el sistema de valores de posición (Power \& Dal Martello, 1997; Seron \& Fayol, 1994). Por una parte, es necesario que el niño aprenda que el orden de las cifras tiene valor significante dentro del código, es decir, que sería incorrecto escribir "61" para denotar "dieciséis" (Dickson, Brown \& Gibson, 1984). En segundo lugar, es necesario que identifique el valor relativo de cada posición dentro del número, es decir, que en el número "16", el 1 representa "diez", mientras que el 6 expresa "seis".

Cuando se comienzan a escribir números de dos cifras también es posible presentar dificultades sintácticas, puesto que el niño tiende a asimilar la simbolización del número en función de lo que ya sabe, como los nombres verbales de los números. Herbert Ginsburg (1977) presenta el caso de una niña, Rebecca, de siete años, que cuando se le pedía que escribiera "veintitrés" escribía "203". A juicio de Ginsburg, este tipo de error es indicativo de una tendencia a transcribir directamente lo que se oye en secuencias de números, sin tomar en cuenta el valor de posición. Así, lo que Rebecca hacía en realidad era escribir dos números que sabía decir: 20 y 3.

El ejemplo de Herbert Ginsburg sirve como pretexto para ilustrar el proceso de aprendizaje del 0 como "no número". Como se observa, Rebecca no tuvo problemas para escribir 20, cuando identificó "veinte" en el "veintitrés" que se dictó. Independientemente de lo equivocado de su respuesta, esto indica una cierta facilidad para manejar el 0 cuando se trata de un número redondo, tal como 10, 200 o 1.000. Esta es una hipótesis sólidamente confirmada en trabajos posteriores. Terezinha Nunes y Peter Bryant (1996) reportan que en muestras de escritura de niños 
británicos, los niños podían escribir correctamente 100 pero no así el número 47; o el número 200 pero no así el número 129, a pesar de que el número 47 es un valor menor y de menor rango numérico que el número 100. El aprendizaje de la escritura de números "redondos" parece llevarse a cabo de manera independiente a su rango.

De acuerdo con Terezinha Nunes y Peter Bryant (1996), es presumible que la memoria juegue un papel en la transcodificación de los números redondos, en la medida en que estos resultan ser particularmente frecuentes, además de escasos (nueve en cada rango numérico). Esta hipótesis sería adecuada para el caso de los números de dos cifras, en la medida en que el niño no posee aún toda la información necesaria para construir un sistema de reglas generativas que sirva para obtener todos los números posibles. Otra interpretación podría anotarse, y es la asignación, al sufijo multiplicativo del sistema verbal "-enta", o "-ente" para el caso del veinte, un signo arábigo particular: el signo " 0 ". Esta segunda hipótesis se basa en la suposición de que, como lo explican Terezinha Nunes y Peter Bryant (1996), los niños tienden a construir un sistema generativo más que un conjunto de accesos específicos a la memoria. Así, el sufijo "-enta/e" corresponde a un cero, y esto más adelante puede generalizarse para el sufijo "-cientos" (en trescientos, por ejemplo) que correspondería a dos ceros (00), y así sucesivamente (Orozco \& Hederich, 2002).

Ahora bien, cuando se introducen numerales de tres y cuatro cifras los problemas sintácticos se incrementan. Terezinha Nunes y Peter Bryant (1996) encuentran en su trabajo con niños británicos que muchos de ellos parecían utilizar dos sistemas: uno para los números de dos dígitos y otro para números de más cifras. Con frecuencia, escribieron correctamente los números redondos $(100,1.000,200)$ y los números de dos dígitos (25 y 47) y se equivocaban en números de más cifras. Richard Power y Maria Felicità dal Martello (1990) encuentran que niños italianos de 7 años transcodifican correctamente números por debajo de 100, pero en números de 3 y 4 dígitos aparecen errores preponderantemente sintácticos (87\%) que consisten, principalmente, en la inserción de ceros extras. Por ejemplo, "trescientos sesenta y cinco" lo escriben 30065. Como lo explicamos con los números de dos cifras, este tipo de errores refleja una intención de traducir literalmente todo lo que se oye, yuxtaponiéndolo. Vemos así que, también en este rango, la traducción literal o "yuxtaposición" es la estrategia más socorrida cuando se intenta escribir números sin tener todavía la regla del valor de posición (Lerner \& Sadovsky, 1994; Power \& Dal Martello, 1990; Seron \& Fayol, 1994; Scheuer, Sinclair, Merlo de Rivas \& Tièche, 2000).
Básicamente, lo que el niño debe aprender es que no todo lo que oye se escribe; que ciertas partes de la cadena hablada —los morfemas "mil", "cientos" y "enta" - no se escriben tal cual sino que sirven como indicadoras de una posición dentro de la expresión arábiga. A fin de escribir correctamente números de hasta cuatro cifras, el niño crea un sistema de cuatro posiciones separando la información correspondiente a los dígitos de la que tiene qué ver con cada una de las posiciones. Por ejemplo, en el número "tres mil cuatrocientos noventa y cinco", el niño identifica los dígitos 3, 4, 9 y 5, y los ubica de acuerdo con la posición indicada por el morfema que lo sigue: de izquierda a derecha: 3 en la primera, 4 en la segunda, 9 en la tercera y 5 en la cuarta.

Durante el desarrollo de la transcodificación de los números de tres y cuatro cifras, hay un elemento adicional que debe aprenderse, se trata de la novedad del 0 intermedio. En este caso, el problema es precisamente el opuesto al que se tiene cuando se aprende el valor de posición. Mientras en este último caso, debe reconocerse que algo que se dice oralmente (los sufijos) no tiene forma gráfica efectiva (solo se marca mediante la posición), en el caso del cero intermedio algo que no se dice oralmente, sí tiene una forma gráfica: el "0". Escribir "trescientos seis", por ejemplo, supone 1) identificar el 3 y el 6; 2) ubicar la posición del 3 en las centenas y la posición del 6 en las unidades y 3) llenar el espacio vacío, el de las decenas, con un 0 . Esta dificultad complementaria es la fuente de errores como escribir "trescientos seis" con la forma 36.

El papel posicional del cero en la representación arábiga de los números es el menos obvio desde la perspectiva de la escritura y es, en consecuencia, el que causa más problemas en el proceso de aprendizaje de la escritura de números (Dickson, Brown \& Gibson, 1984; Haas, 1996; Nunes \& Bryant, 1996). Desde el punto de vista del niño, está aprendiendo que el 0 es una especie de dígito "no dígito", en la medida en que tiene la forma de un dígito pero no corresponde a ninguna cantidad. Como ya lo hemos explicado, cuando el 0 se encuentra en posiciones finales, y en particular en números redondos, su presencia puede asimilarse a la de un sufijo multiplicativo específico, pero en el caso en que el cero es intermedio debe reconocerse su verdadero valor sintáctico, es decir, un marcador de ausencia de cantidad en la posición correspondiente.

Finalmente, cuando se llega a los rangos de números de cinco cifras o más, se produce una complicación adicional. En este nivel, el niño debe advertir que: 1) el sistema posicional que ha aprendido no posee cuatro sino tres posiciones y 2) tal sistema tiene un potencial recursivo infinito.

Con respecto al primer punto, el niño debe aprender que, superada la barrera de las tres posiciones 
iniciales, el valor multiplicativo del morfema "mil" cubre no solo la cuarta posición, sino todas las posiciones ocupadas a la izquierda. Curiosamente, esto no lo puede advertir con la simple aparición del morfema "mil" (para números de cuatro cifras), sino por contraste, con el hecho de que el morfema que marcaría la quinta posición en su sistema no es un morfema nuevo, como cabría esperarse, sino el ya conocido morfema "-enta/e". Así, en un número como "cuarenta y cinco mil novecientos ochenta y siete", la partícula "mil" no está indicando una cuarta posición sino que es una marca de separación entre una serie de dos posiciones a la izquierda, de las tres que le siguen a la derecha². Al respecto, Linda Dickson, Margaret Brown y Olwen Gibson (1984) señalan que un error frecuente durante la lectura en voz alta de números de cinco cifras es que los niños tienden a sufijar cada dígito con un morfema diferente. Así, el número 23456 es leído como "dos millones tres mil cuatrocientos cincuenta y seis". Este tipo de errores de lectura es un indicador de que se ha construido un sistema de cinco posiciones, donde cada posición está marcada por un morfema específico.

Junto con la nueva comprensión del "verdadero" rango multiplicativo del morfema "mil" aparece la segunda constatación: que la regla de las tres posiciones puede repetirse hacia la izquierda todas las veces que se quiera. En este carácter recursivo está el potencial generativo del sistema verbal, como código que permite decir cualquier número posible.

A manera de ejemplo, observemos cómo se produciría la transcodificación de la expresión "Novecientos cuarenta y cinco mil trescientos ochenta y siete" En primer lugar, se diferencian en esta expresión las bases léxicas que denotan dígitos, de los morfemas multiplicativos:

$=[9$-cientos 4-enta y 5$]$ mil 3-cientos 8-enta y 7

Los sufijos "-ciento" y "-enta" se reconocen como operadores multiplicativos: $x 100$ y x10, respectivamente, que actúan sobre el dígito previamente mencionado. Por su parte, el morfema "mil" se identifica también como un operador multiplicativo (x1000) cuya función sintáctica afecta, no solo al dígito previo, sino a los tres dígitos anteriores:

$$
=[9 \times 100+4 \times 10+5] \times 1000+3 \times 100+8 \times 10+7
$$

Con la aplicación de la regla del valor de posición y la del campo de acción del morfema "mil", se construye entonces la secuencia:

$$
=[900+40+5] \times 1000+[300+80+7]=945387
$$

Así, cuando la expresión numérica supera la barrera de las centenas, el proceso de marcación morfosintáctica es similar (morfemas) pero su régimen (es decir, su rango de influencia) se amplía a toda la expresión que precede al sufijo.

Al igual que con los números de tres y cuatro cifras, una de las principales fuentes de error en la escritura de números de cinco o más cifras es cuando en la expresión numérica verbal hablada no aparecen unidades en un momento de la cadena fonética. En este caso, lo que debería pasar es que simplemente la información correspondiente al dígito en ese

2 Las expresiones numéricas verbales escritas indican esto mediante la diferencia entre el morfema libre "mil", que opera como una palabra, y los morfemas ligados "-cientos" y "-enta" que operan como sufijos derivativos. 
orden sea omitida junto con la información sintáctica correspondiente a ese orden. Por ejemplo:

"Novecientos cuarenta y cinco mil ochenta y siete"

$=[9$-cientos 4-enta y 5] mil $\theta$-cientos 8 -enta y $7=945087$

La omisión de un orden en la expresión verbal hablada genera errores tanto de inclusión de secuencias llenas de ceros, en un intento, una vez más, de escribir lo que se oye, como de reducción de la cadena gráfica, como 945.87, en una evidente destrucción del marco sintáctico de tres posiciones.

Una posible explicación para este último tipo de errores es una particularidad del sistema arábigo que no hemos comentado todavía. Si bien los números se construyen de manera creciente (de cantidades menores a la derecha a cantidades mayores a la izquierda), estos se escriben y se leen en dirección decreciente (de cantidades mayores a la izquierda, a cantidades menores a la derecha). Este cambio de dirección entre lo que se comprende y lo que efectivamente se produce no es percibido fácilmente. Por ejemplo, para el número 2806443597, debemos identificar valores de posición comenzando con las unidades y procediendo de derecha a izquierda en orden creciente de magnitud. Así, primero "leemos" el número de derecha a izquierda y luego lo pronunciamos de izquierda a derecha (Haas, 1996).

En esta descripción cognitiva del desarrollo de la transcodificación en niños escolarizados sin necesidades educativas especiales, hemos intentado mostrar los requisitos de aprendizaje que cada rango de números supone $y$, junto con ello, las principales fuentes de facilidad o dificultad que, para cada rango de números, genera su escritura por parte del sujeto que la está aprendiendo. Examinemos ahora nuestros resultados para cada grupo de edad y grado escolar, en cada rango numérico.

\section{Método}

\section{Diseño de la investigación}

Esta investigación puede ser considerada como un estudio observacional transversal, en que se registran y analizan los desempeños de sujetos diferenciados por grado cursado, y, por tanto, por edad, frente a una tarea de dictado de números.

En la medida en que intentamos examinar el proceso de desarrollo de la tarea de transcodificación de numerales verbales a formato arábigo, lo ideal habría sido seguir el desarrollo de las ejecuciones de los mismos sujetos frente a la tarea durante un tiempo lo suficientemente largo como para dar cuenta de cambios conductuales; esto es, lo ideal habría sido un diseño longitudinal. No es este nuestro caso, por lo que las inferencias sobre el desarrollo deben hacerse sobre la base de las ejecuciones de grupos diferentes, estratificados por la edad y el grado cursado. Por las limitaciones de este diseño, consideramos que el estudio tiene un carácter más bien exploratorio que confirmatorio.

\section{Muestra}

El estudio se llevó a cabo con una muestra de 118 estudiantes de los dos géneros de los grados $1^{\circ}, 2^{\circ}, 3^{\circ}$ y $4^{\circ}$ de educación básica primaria de una institución educativa oficial localizada en Bogotá, Colombia, con edades comprendidas entre los 7 y los 11 años. Los promedios de edad para cada grado, con sus respectivas desviaciones estándar se presentan en la tabla 2. 
Tabla 2

Muestra efectiva

\begin{tabular}{c|c|c|c}
\hline Grado & $\begin{array}{c}\text { Edad } \\
\text { promedio }\end{array}$ & $\begin{array}{c}\text { Desviación } \\
\text { estándar }\end{array}$ & Número \\
\hline 1 & 7,44 &, 26 & 28 \\
\hline 2 & 8,24 &, 33 & 29 \\
\hline 3 & 9,28 &, 36 & 31 \\
\hline 4 & 10,57 &, 51 & 30 \\
\hline Total & 8,89 & 1,21 & 118 \\
\hline
\end{tabular}

Fuente: elaboración propia

Instrumento: prueba de dictado de números

El análisis de la tarea de transcodificación de numerales verbales presentada en un punto anterior mostró que las principales características de numeral que podrían facilitar, o dificultar, su transcodificación son: 1) su longitud (a mayor longitud del numeral arábigo, mayor dificultad); 2) la presencia de ceros intermedios en la expresión arábiga del numeral (que dificulta mucho más este tipo de numerales; y 3) el carácter particular de los números "redondos", cuya escritura parece ser bastante precoz en cada orden.

Sobre la base de este análisis, se elaboró una lista de numerales con una longitud entre dos y seis dígitos, de acuerdo con las siguientes reglas:

1. No se repiten dígitos. Ningún numeral incluye dígitos iguales en posiciones diferentes.

2. El rango de los numerales dictados varía en función del grado que cursan los niños, de acuerdo con la tabla 3:

Tabla 3

Rangos numéricos en cada grado

\begin{tabular}{c|c|c|c|c|c}
\hline Grado & \multicolumn{5}{|c}{ Número de dígitos } \\
\hline & 2 & 3 & 4 & 5 & 6 \\
\hline 1 & $\mathrm{x}$ & $\mathrm{x}$ & & & \\
\hline 2 & & $\mathrm{x}$ & $\mathrm{x}$ & $\mathrm{x}$ & \\
\hline 3 & & & $\mathrm{x}$ & $\mathrm{x}$ & \\
\hline 4 & & & & $\mathrm{x}$ & $\mathrm{x}$ \\
\hline
\end{tabular}

Fuente: elaboración propia

3. En cada rango, se incluyeron numerales que contenían todas las posibles combinaciones de ceros, en todas las posibles posiciones.

4. Para los numerales dictados a los niños de primer grado, cada tipo de numeral fue repetido, modificando los dígitos que componen su expresión arábiga, de forma que se dictó un número compuesto enteramente por dígitos menores a 5 y otro compuesto por dígitos mayores a 5. Esto se hizo para determinar si había diferencias entre estos dos rangos de números (los menores o iguales a 5 y los mayores que 5). El total de los numerales seleccionados se mezcló de forma aleatoria para cada grado.

Una vez se tuvo la lista de numerales, se procedió al dictado de los números. El dictado es una tarea de papel y lápiz, de aplicación grupal y resolución individual, en la que se enuncia en formato verbal hablado cada uno de los numerales, cuya versión en formato arábigo los niños deben escribir en un cuadernillo diseñado para ello. Los numerales fueron dictados "de corrido", es decir, sin hacer pausas artificiales en su enunciación. En la medida en que no estábamos interesados en los errores léxicos, cada numeral fue dictado tantas veces como lo requirieron los niños.

\section{Procesamiento y análisis}

Las muestras de escritura de los niños fueron recogidas en 2001 y transcritas literalmente a una base de datos en la que los casos son cada uno de los sujetos que respondieron y las variables son cada uno de los numerales dictados. Esta base fue inicialmente analizada para identificar las producciones erróneas. Luego, las producciones erróneas fueron clasificadas según si correspondían a errores léxicos o a errores sintácticos. Cuando el error era únicamente el cambio de uno o varios dígitos por otros, sin que se afectara la estructura sintáctica del numeral, este se clasificó como error léxico. Por su parte, cuando el error era de variación de la longitud de la cadena de dígitos (por decremento o por incremento), este era clasificado como error sintáctico. La posición del punto, en el caso en que hubiera alguno en una producción dada, fue ignorada como fuente de error sintáctico.

Con esta base de datos inicial, se construyó otra en la que cada caso constituye un numeral específico, junto con los índices de dificultad empírica del número para cada grado en que fue preguntado. Suponemos que la complejidad de un ítem está dada por su nivel empírico de dificultad sintáctica, es decir, por el porcentaje de respuestas sintácticamente incorrectas frente al ítem. Para el análisis se consideraron como respuestas correctas, tanto los casos de identificación total entre el número dictado y el número escrito, como los casos en que se cometieron únicamente errores léxicos o de posición del punto.

\section{Resultados y discusión}

La figura 1 presenta los promedios de logro sintáctico en la transcodificación (entendidos como 
porcentaje de respuestas sintácticamente correctas) por grado escolar y número de dígitos del numeral.

Figura 1

Logro sintáctico

Logro sintáctico por número de cifras del numeral en cada grado

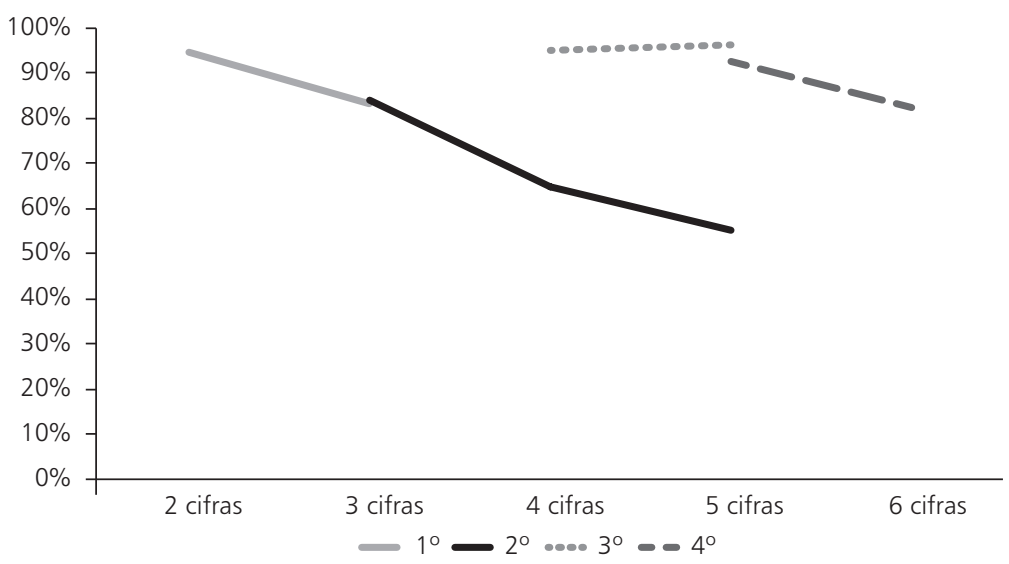

Fuente: elaboración propia

Tal como se observa, los niños de primer grado muestran un logro casi total en numerales de dos cifras (en promedio, 95\% de producciones correctas). Los números de dos cifras presentados a los niños de primer grado fueron seis: $40,45,50,80,89$ y 90 . Si bien no hay mayores diferencias en los niveles de logro entre estos numerales, puede identificarse que los que resultan más difíciles son "89" (86\%) y "45" (94\%), que en este caso son los números no redondos.

En números de tres cifras, los porcentajes de logro sintáctico de los niños de primer grado descienden levemente, hasta un promedio global de $83 \%$ (figura 1). Es interesante observar en detalle las diferencias entre los niveles de dificultad de los números particulares. Tal como se observa en la tabla anexa, los números que resultaron ser más fáciles fueron "400", "700", "405" y "709" (logros de más del 95\%), mientras que los números más difíciles fueron "790" (64\%), seguido de "453" y "796" (67\%).

Varios puntos deben señalarse en estos resultados. Primero, volvemos a verificar el hecho de que los números redondos parecen ser más sencillos para su transcodificación. Segundo, surge el hecho, que ya habíamos verificado en el orden anterior, de que los números "completos", sin ceros intermedios, parecen presentar altos niveles de dificultad para su transcodificación. Tercero, aparece un hecho sumamente curioso: la baja dificultad en los numerales 405 y 709, los dos numerales con ceros intermedios en su expresión arábiga, que podría pensarse asociada más bien con altos niveles de dificultad.

El hecho de que los dos numerales con ceros intermedios resulten tan particularmente fáciles para los niños de primer grado resulta notable. Tal como lo hemos argumentado antes, la presencia de un 0 intermedio en un numeral arábigo no está indicada explícitamente en su correspondiente forma verbal, por lo cual podría suponerse que se aumenta la complejidad de la tarea en la medida en que se requeriría la elaboración de una inferencia. Los resultados, sin embargo, contradicen este supuesto.

Los niños de segundo grado resolvieron la transcodificación de numerales de tres, cuatro y cinco cifras. En números de tres cifras, estos 
niños muestran porcentajes de logro sintáctico equivalentes a los de primer grado (84\%). Como cabe prever, en números de cuatro cifras, el logro sintáctico de los niños de segundo grado desciende a menos del 65\%, y en números de cinco cifras vuelve a descender (55\% de producciones correctas en su sintaxis). Esto es consistente con la idea de que a medida que aumentamos en rango numérico, introducimos nuevas complejidades sintácticas en el sistema.

Resulta curioso observar una aparente detención en el proceso de desarrollo entre el primero y el segundo grado, marcada por porcentajes similares de logro en numerales de tres cifras.

La figura 2 muestra los niveles de logro sintáctico de los niños de primero y segundo grado frente a cada numeral. Como hemos mencionado, los niños de segundo grado resolvieron dictados de números de tres, cuatro y cinco cifras y sus porcentajes de logro sintáctico son equivalentes a los obtenidos en primer grado. En los números de tres cifras, resulta relevante destacar varios puntos. Primero, de nuevo para este grado se verifica que los números redondos son relativamente fáciles. Segundo, los números 796, 790 y 800 que resultaban difíciles para primer grado, ahora resultan más fáciles. Esto parece apoyar la idea de que los niños de primer grado encuentran más difíciles los números compuestos por dígitos mayores a 5. Tercero, los numerales 405 y 709, que resultaban muy fáciles para primer grado, resultan ahora relativamente difíciles. Este último es, sin duda, el resultado más notable, por lo que lo elaboraremos con mayor detalle.

Figura 2

Niveles de logro sintáctico de los niños de $1^{\circ}$ y $2^{\circ}$ grados

frente a cada numeral dictado

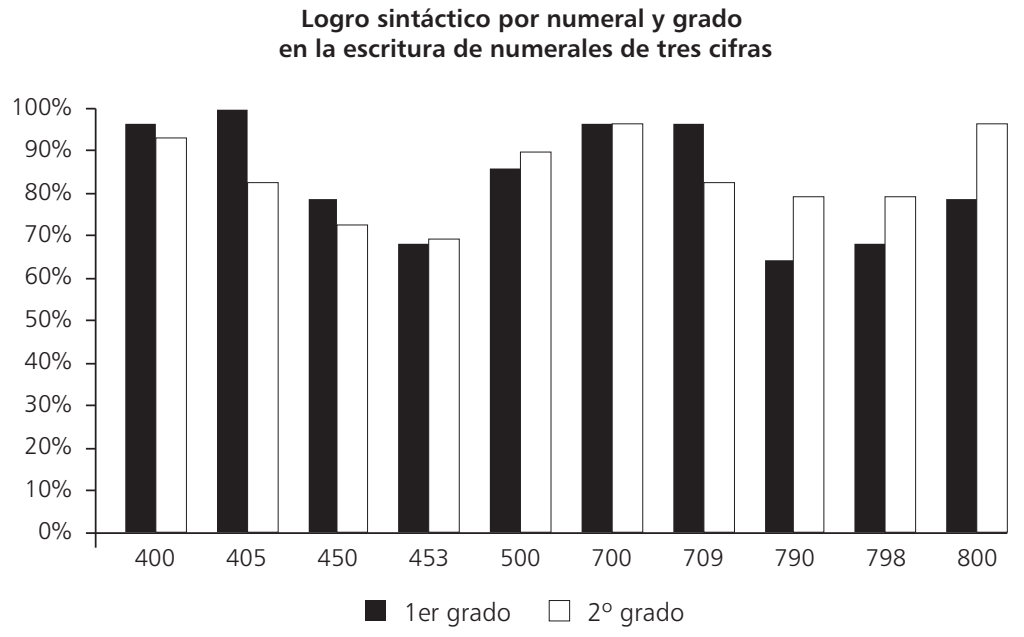

Fuente: elaboración propia

De acuerdo con las hipótesis de Terezinha Nunes y Peter Bryant (1996), los números redondos pueden ser fácilmente transcodificados, en gracia a su frecuencia y a su escasa cantidad, por un proceso de recuperación en la memoria. Esta hipótesis no parece muy plausible en el caso de los números con ceros intermedios, cuya frecuencia es mucho menor y su cantidad mayor (habría 99 para numerales de tres dígitos). Así, debemos acudir a la idea de que los niños construyen un sistema de generación específico para estos números, posiblemente a partir de lo que conocen sobre números redondos en ese orden, que indica que el sufijo "-cientos" 
se codifica por medio de dos ceros. Ahora, como sabemos, el sufijo "-cientos" indicaría dos ceros, los que en una traducción literal produciría, por ejemplo, el numeral 4005 por 405. Bien, nuestra hipótesis es que, efectivamente, los niños de primer grado cometen posiblemente un tipo de error que antes hemos descrito como "compactación" (Orozco \& Hederich, 2002). En otras palabras, en realidad estos niños estaban produciendo la expresión 4005, con supresión de un cero, lo que en este caso los lleva a la respuesta correcta (405). Así, estos niños tienen un procedimiento que les permite producir la respuesta correcta, si bien tal procedimiento no responde al marco sintáctico convencional del formato arábigo.

Por su parte, en los niños de segundo grado esta regla de codificación de sufijos por medio de ceros parece haber desaparecido a favor de la construcción de una regla generativa más general, la regla del valor de posición. En el momento en que los sufijos ya no se traducen por medio de ceros sino por medio de posiciones, el cero intermedio se convierte en un problema. Parecería que los niños de segundo, si bien ya han construido la regla de valor de posición, como lo atestigua el hecho de que pueden transcribir con propiedad números como 796, todavía tienen problemas con la regla de uso del cero como marcador de no cantidad en el orden de las decenas.

Este problema se acentúa en números de cuatro cifras, en los cuales el logro de segundo grado desciende de nuevo de manera brusca hasta situarse, en promedio, en 65\%. Es interesante observar las características de los numerales que parecen asociadas con su complejidad. Como en los rangos anteriores, los números redondos (2000, 3000, 8000 y 9000) son bastante más fáciles que los otros. Los números más difíciles resultan ser 2040, 2053, 8009, 8090 y 8096: en todos los casos, estos números presentan un " 0 " en la posición de las centenas.

Estamos aquí frente al mismo problema observado con el cero intermedio en los números de tres cifras. Cuando el niño escucha "dos mil cincuenta y tres" escucha tres dígitos: "dos", "cinco" y "tres". Sin embargo, también escucha el morfema "mil" y no escucha el morfema "-cientos". Así, si utiliza la regla que ya sabe, puede intentar marcar esa partícula "mil" por medio de un punto y escribir, 2.53 (7 casos, 50\% de las producciones incorrectas); puede completar las posiciones totales del número añadiendo un cero al final (2.530); puede ignorar (hacerse el que no oyó) el morfema "mil" y escribir 253; o incluso podría regresar al nivel más literal de la traducción suponiendo que ha oído varios números y escribir 2000.503 o alguna variante en la que se compactan algunos ceros (20053, por ejemplo). En todos los casos, el problema parece surgir de la ausencia de unidades en la posición de las centenas, y los diferentes errores han sido previamente descritos en nuestro trabajo anterior. A manera de ilustración, la tabla 4 muestra la frecuencia de las producciones de los niños de segundo grado frente a este número.

Tabla 4

Frecuencia de las producciones de segundo grado frente al dictado del numeral "dos mil cincuenta y tres"

\begin{tabular}{|c|c|c|c|}
\hline & Producción & Frecuencia & (\%) \\
\hline \multirow[t]{2}{*}{$\begin{array}{l}\text { Producciones } \\
\text { correctas }\end{array}$} & 2.053 & 11 & 37,93 \\
\hline & 2053 & 4 & 13,79 \\
\hline Subtotal & & 15 & 51,72 \\
\hline \multirow[t]{8}{*}{$\begin{array}{l}\text { Errores } \\
\text { sintácticos }\end{array}$} & 2.53 & 7 & 24,14 \\
\hline & 253 & 1 & 3,45 \\
\hline & 2.530 & 1 & 3,45 \\
\hline & 2000.53 & 1 & 3,45 \\
\hline & 200.057 & 1 & 3,45 \\
\hline & 200.503 & 1 & 3,45 \\
\hline & 20.053 & 1 & 3,45 \\
\hline & 200.53 & 1 & 3,45 \\
\hline Subtotal & & 14 & 48,28 \\
\hline Total & & 29 & 100 \\
\hline
\end{tabular}

Fuente: elaboración propia

Cuando los niños de segundo grado deben escribir numerales de cinco cifras, las posibilidades de error sintáctico se incrementan, lo cual aparece indicado por una disminución global en el promedio de logro sintáctico, de $64 \%$ en números de cuatro cifras, a $55 \%$ en numerales de cinco cifras. Un análisis de los numerales específicos de mayor o menor complejidad sintáctica en este rango no muestra grandes cambios frente a lo ya expuesto para rangos inferiores: 1) los números redondos siguen mostrando escasos errores, al igual que los numerales sin ceros intermedios, y 2) los numerales con ceros intermedios, en la posición de las centenas $(82045,80045,82049,82004 \ldots)$, siguen mostrando el mayor índice de errores sintácticos (entre $35 \%$ y $45 \%$ de aciertos).

Los niños de tercer grado muestran un aumento muy considerable de logro sintáctico frente a los de segundo grado en números de cuatro y cinco cifras, que llegan casi hasta el logro sintáctico completo (95\% para cuatro cifras y $96 \%$ para cinco). Puede entonces considerarse que estos niños ya manejan la sintaxis del sistema arábigo, al menos en estos rangos numéricos. Este resultado coincide con el reportado por Kara S. Sullivan, Paul Macaruso y Scott Sokol (1996) y 
Marie-Pascale Nöel y Eva Turconi (1999), quienes plantean que se requieren tres años de escolaridad para alcanzar el dominio completo de la transcodificación de números de cuatro cifras.

Un análisis detallado de los numerales con mayores y menores niveles de dificultad en este grado muestra las mismas tendencias encontradas en segundo grado: 1) los nodos resultan significativamente más sencillos; y 2) los numerales con cero en la posición de las centenas resultan relativamente más complejos.

En general, y para todos los numerales, los niños de tercer grado muestran mejores porcentajes de logro sintáctico que los de segundo. Las menores diferencias entre los grados se presentan en los numerales "redondos", bastante fáciles para los dos grados, y las mayores diferencias en los numerales con ceros en la posición de las centenas.

Los niños de cuarto grado muestran porcentajes de logro sintáctico similares a los de tercer grado en números de cinco cifras, si bien debe notarse que estos resultan levemente menores (92\% y 91\%, respectivamente). Al aumentar a seis el número de cifras para niños de cuarto grado, de nuevo se observa un incremento de la dificultad sintáctica, representado por un descenso del logro sintáctico hasta situarse en $82 \%$ y $84 \%$, respectivamente. Este fenómeno no amerita más explicación que la del aumento de la complejidad sintáctica de la tarea, que ya ha sido explicado.

De nuevo, como ya lo habíamos verificado en el paso entre primero y segundo grados, encontramos un leve descenso en la eficacia conductual de la tarea. Los niños de tercer grado muestran mejor eficacia que los de cuarto. Si admitimos el supuesto de la comparabilidad entre estos grupos. ¿Qué explica este descenso? Examinaremos, esto en detalle:

La figura 3 representa las diferencias entre los porcentajes de logro sintáctico de los niños de cuarto grado y los de tercer grado para cada uno de los numerales dictados y muestra que la diferencia de porcentajes en el logro de los niños de cuarto grado resulta inferior al obtenido por los de tercer grado.

Figura 3

Diferencias en porcentajes de logro sintáctico entre $3^{\circ}$ y $4^{\circ}$ grados

Logro sintáctico por numeral y grado en la escritura de numerales de cinco cifras

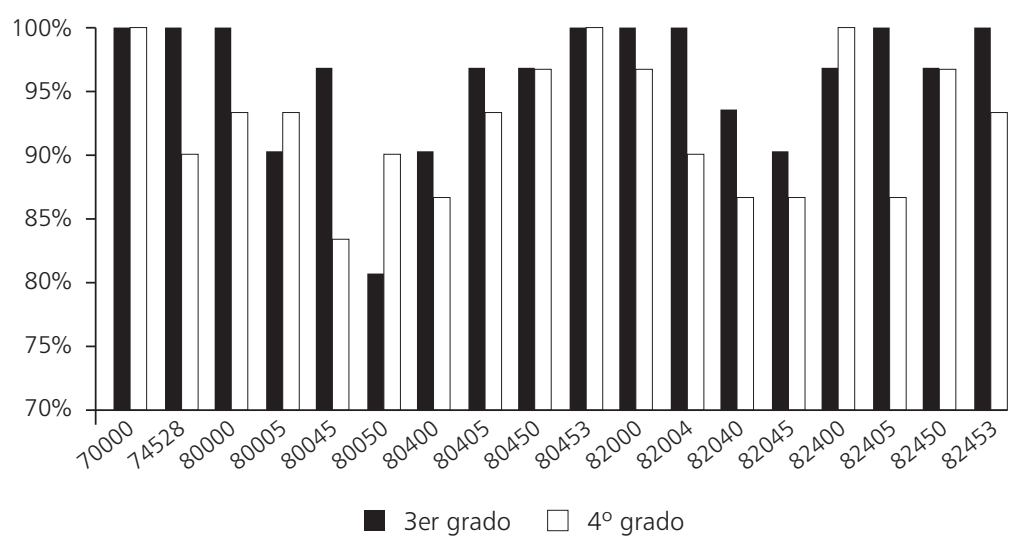

Fuente: elaboración propia

Al examinar la significación de las diferencias, mediante la aplicación de pruebas T de Student sobre muestras independientes, las únicas diferencias que resultan significativas a favor de los niños de tercer grado se dan 
en el numeral completo sin ceros intermedios 74528 $(t=-2,449, p=0,017)$ y en los numerales 80045 $(t=2,095, p=0,40)$ y $82405(t=2,148, p=0,036)$. Si relajamos la exigencia del nivel de significación hasta 0,1 aparecen, también a favor de los niños de tercer grado, los numerales $82004(t=1,779, p=0,08) y$, de nuevo, un numeral completo sin ceros intermedios: 82453 ( $t=1,779, p=0,08)$.

De acuerdo con el programa curricular colombiano, durante el tercer grado, los niños deben lograr el manejo completo del sistema de numeración decimal para los números enteros. Este requerimiento parece ser cumplido, como lo atestiguan en nuestro caso los logros mostrados por los niños de tercer grado en numerales de cuatro y cinco cifras. Ahora, si podemos suponer que estos mismos niveles fueron logrados, en su momento, por los niños de cuarto grado, asistimos en este caso a una especie de regresión, de descenso en la eficacia conductual de los niños en la tarea de la transcodificación.

Habida cuenta de lo anterior, nuestras hipótesis podrían explicar los descensos en las eficacias conductuales, como momentos específicos en los cuales los niños están incorporando marcos sintácticos más amplios. En el caso de los niños de cuarto grado, sabemos que el desarrollo de los programas curriculares se centra en la generalización del sistema de numeración de forma que permita la expresión de números racionales. Por otro lado, podríamos suponer - aunque no podemos describir este proceso en detalle- que el sistema de numeración verbal se está generalizando a números de más de siete cifras en los que aparece por primera vez la partícula "millones".

\section{Conclusiones}

Los resultados presentados permiten sostener la hipótesis de que, frente a cada rango numérico, el niño construye un sistema estable de generación de numerales que se desestabiliza con la llegada del siguiente orden numérico.

Este sistema de generación tiene rasgos específicos para cada grado y rango de edad. Los niños de primer grado parecen asimilar los sufijos que indican operadores multiplicativos a la adición de ceros en el formato arábigo. Eso les permite generar las expresiones arábigas correctas en numerales de dos y tres cifras, y particularmente si en estos numerales aparecen ceros intermedios, pero fallan en la transcodificación de los numerales "completos", sin ceros intermedios. Posiblemente, estemos asistiendo a una situación en la que el sistema completo se ha desestabilizado para incorporar las reglas sintácticas requeridas para las tres posiciones, por lo que se ha disminuido la eficacia conductual frente a números previamente dominados. Este fenómeno ha sido documentado previamente dentro del desarrollo cognitivo (Karmiloff-Smith, 1994).

Los niños de segundo grado, por el contrario, parecen haber iniciado la interpretación adecuada de los sufijos multiplicativos hacia la definición de una posición específica en la cadena arábiga, pero entonces se topan con dificultades en los casos de los numerales en que los no se indican, de forma explícita, algunas cantidades en algunas posiciones (numerales con ceros intermedios). Esta dificultad se mantiene y se hace mucho más evidente en numerales de cuatro y cinco cifras.

Los niños de tercer grado, por su parte, ya parecen haber superado, en la mayoría de los casos, estas dificultades. Solo alcanza a verse una cierta dificultad relativa en los casos en que la ausencia de cantidades se da en el orden de las centenas, en numerales de cuatro y cinco cifras. Esto indica que la regla que se aprende en último lugar, y que representa el obstáculo final para el aprendizaje completo de la transcodificación, es el hecho de que la partícula "mil" que aparece en estos rangos, afecta, no solo al número que le precede (cuatro mil), sino a los anteriores (cincuenta y cuatro mil).

Suponemos que los niños que, en el momento de la toma de muestras cursaban el grado cuarto, lograron el mismo resultado evidenciado por los de tercer grado cuando estaban en ese curso. En el momento de la toma de muestras, sin embargo, su atención debe estar centrada en la generalización del sistema de numeración a numerales de siete o más posiciones y hacia numerales no enteros y específicamente números racionales. Por esta razón, los marcos sintácticos construidos hasta entonces, y que daban cuenta de numerales de seis o menos cifras, deben ser momentáneamente desestabilizados, lo cual explicaría su leve descenso en la eficacia conductual frente a la tarea. Esta última hipótesis, sin embargo, debe ser confirmada en trabajos posteriores que examinen en detalle la construcción de marcos sintácticos para estos nuevos números.

En conclusión, podemos postular una secuencia definida para el aprendizaje de las reglas sintácticas del sistema de notación decimal que inician con la memorización de los números redondos de dos y tres cifras y continúa con la asimilación de sufijos multiplicativos. Esto permite a los niños de primero y segundo grados tener desempeños correctos en numerales de tres cifras cuando estos no tienen ceros intermedios, pero faIlan cuando no hay mención explícita de una posición. Esta dificultad parece ya superada en niños de tercer grado. Solo algunas dificultades aisladas permanecen en estos niños, relacionadas con números de cuatro y cinco cifras que no tienen cantidades en el orden de 
las centenas. Estas dificultades no alcanzan a quedar completamente solucionadas en los niños de cuarto grado, pues la eficacia conductual de la transcodificación, incluso, disminuye levemente frente a algunos números, seguramente como resultado de nuevos aprendizajes numéricos.

En general, se puede apoyar la hipótesis de que el desarrollo de la transcodificación sigue un proceso discontinuo marcado de ascensos y descensos bruscos de la eficacia conductual de los niños frente a la tarea, a medida que se van incorporando numerales cada vez mayores y se van construyendo marcos sintácticos más generales. Los aprendizajes posteriores desestabilizan los sistemas previos en formas que aún no se comprenden del todo.

\section{Sobre los autores}

Christian Hederich-Martínez es matemático. Magíster en desarrollo educativo y social y doctor en psicología. Profesor e investigador, Universidad Pedagógica Nacional, Bogotá, Colombia y líder del grupo de investigación en Estilos Cognitivos. Ha publicado nueve libros y más de 50 artículos sobre psicología educativa.

Ángela Camargo-Uribe es licenciada en Lenguas. Magíster en lingüística general, doctora en educación. Profesora e investigadora, Universidad Pedagógica Nacional. Miembro del grupo de investigación en Estilos Cognitivos. Editora de la Revista Folios, Revista de la Facultad de Humanidades de la Universidad Pedagógica Nacional.

Angélica Avalo-Azcárate es psicóloga, Universidad del Valle. Estudiante de la maestría en educación, Universidad Pedagógica Nacional.

\section{Referencias}

Barrouillet, P.; Camos, V.; Perruchet, P. \& Seron, X. (2004). ADAPT: A Developmental, Asemantic, and Procedural Model for Transcoding From Verbal to Arabic Numerals. Psychological Review, 111 (2), 368-394. Disponible en: http://www.researchgate.net/publication/8635508_ ADAPT_A_Developmental_Asemantic_and_Procedural_Model_for_ Transcoding_From_Verbal_to_Arabic_Numerals

Camos, V. (2008). Low Working Memory Capacity Impedes both Efficiency and Learning of Number Transcoding in Children. Journal of Experimental Child Psychology, 99, 37-57. Disponible en: http://www. researchgate.net/publication/5985453_Low_working_memory_ capacity_impedes_both_efficiency_and_learning_of_number transcoding_in_children

Campbell, J. I. D. \& Clark, J. M. (1988). An Encoding-Complex View of Cognitive Number Processing: Comments on McCloskey, Sokol \& Goodman (1986). Journal of Experimental Psychology General, 117 (2), 204-214.

Campbell, J. I. D. \& Clark, J. M. (1992). Cognitive Number Processing: An Encoding-Complex Perspective. En James I. D. Campbell (ed.). The Nature and Origins of Mathematical Skills, 457-492. Amsterdam: Elsevier.

Cipolotti, L. \& Butterworth, B. (1995). Toward a Multiroute Model of Number Processing: Impaired Number Transcoding with Preserved Calculation Skills. Journal Experimental Psychology: General, 124 (4), 375-390.

Cohen, L. \& Dehaene, S. (1995). Number Processing in Pure Alexia. The Effect of Hemispheric Asymmetries and Tasks Demands. Neurocase, 1, 
121-138. Disponible en: http://www.unicog.org/publications/ CohenDehaene_NumberProcessingPureAlexia_Neurocase1995.pdf

Dehaene, S. (1992). Varieties of Numerical Abilities. Cognition, 44 (1-2) 1-33. Disponible en: http://www.unicog.org/publications/Dehaene_ VarietiesOfNumericalAbilities_Cognition1992.pdf

Dehaene, S. \& Cohen, L. (1995). Towards an Anatomical and Functional Model of Number Processing. Mathematical Cognition, 1, 83-120. Disponible en: http://www.unicog.org/publications/DehaeneCohen_ TripleCodeModelNumberProcessing_MathCognition1995.pdf

Deloche, G. \& Seron, X. (1982a). From One to 1: An Analysis of a Transcoding Process by Means of Neuropsychological Data. Cognition, 12 (2), 119-149.

Deloche, G. \& Seron, X. (1982b). From Three to 3: A Differential Analysis of Skills in Transcoding Quantities between Patients with Broca's and Wernicke's Aphasia. Brain, 105 (4), 719-733. Disponible en: http:// brain.oxfordjournals.org/content/105/4/719

Deloche, G. \& Seron, X. (1987). Numerical Transcoding: A General Production Model. En Gérard Deloche \& Xavier Seron (eds.). Mathematical disabilities: A Cognitive Neuropsychological Perspective, 137-170. Hillsdale, New Jersey: Erlbaum.

Dickson, L.; Brown, M. \& Gibson, O. (1984). El aprendizaje de las matemáticas. Barcelona: Labor, Ministerio de Educación y Ciencia, MEC.

Ginsburg, H. (1977) Children's Arithmetic: the Learning Process. New York: van Nostrand.

Haas, W. (1996). Sobre la escritura de los números. En Nina Catach (ed.). Hacia una teoría de la lengua escrita, 257-270. México: Gedisa.

Karmiloff-Smith, A. (1994). Más allá de la modularidad: la ciencia cognitiva desde la perspectiva del desarrollo. Madrid: Alianza.

Lerner, D. \& Sadovsky, P. (1994). El sistema de numeración: un problema didáctico. En Cecilia Parra \& Irma Saiz (comp.). Didáctica de las matemáticas, 95-184. Buenos Aires: Paidós.

McCloskey, M. (1992). Cognitive Mechanisms in Numerical Processing. Evidence from Adquired Dyscalculia. Cognition, 44 (1-2), 107-157.

McCloskey, M.; Aliminosa, D. \& Sokol, S. M. (1991). Facts, Rules, and Procedures in Normal Calculation: Evidence from Multiple Single-Patient Studies of Impaired Arithmetic Fact Retrieval. Brain and Cognition, $17(2), 154-203$.

Moura, R.; Wood, G.; Pinheiro-Chagas, P.; Lonnemann, J., Krinzinger, H., Willmes, K. \& Haase, V. G. (2013). Transcoding Abilities in Typical and Atypical Mathematics Achievers: the Role of Working Memory and Procedural and Lexical Competencies. Journal of Experimental Child Psychology, 116 (3), 707-727. DOI: 10.1016/j.jecp.2013.07.008

Noël, M. P. \& Seron, X. (1992). Arabic Number Reading Deficit: A Single Case Study or When 236 is Read (2306) and Judged Superior to 1258. Cognitive Neuropsychology, 10 (4), 317-339. Disponible en: https://www. researchgate.net/publication/247514678_Arabic_Number_Reading_ Deficit_A_Single_Case_Study_or_When_236_Is_Read_\%282306\%29_ and_Judged_Superior_to_1258

Noël, M. P. \& Turconi, E. (1999). Assessing Number Transcoding in Children. European Review of Applied Psychology, 49 (4), 295-302.

Nuerk, H. C.; Weger, U. \& Willmes, K. (2005). Language Effects in Magnitude Comparison: Small, but Not Irrelevant. Brain and Language, 92 (3), 262-277. 
Nunes, T. \& Bryant, P. (1996). Comprensión de los sistemas de numeración. Las matemáticas y su aplicación: la perspectiva del niño. México: Siglo XXI Editores. Segunda Edición en Español (1998).

Orozco, M.; Guerrero, D. \& Otálora, Y. (2007). Los errores sintácticos al escribir numerales en rango superior. Infancia y Aprendizaje: Journal for the Study of Education and Development, 30 (2), 147-162.

Orozco, M. \& Hederich, C. (1998). Construcción de la operación multiplicativa y del sistema notacional en base 10: una relación posible. Informe final de la investigación. Cali: Universidad del Valle, Colciencias. Manuscrito sin publicar.

Orozco, M. \& Hederich, C. (2002). Errores de los niños al escribir numerales dictados. Disponible en: http://paginasweb.univalle.edu. $\mathrm{co} / \sim$ cognitiva/\#

Otálora, Y. \& Orozco, M. (2006). ¿Por qué 7345 se lee como "setenta y tres cuarenta y cinco"? Revista Latinoamericana de Investigación en Matemática Educativa, 9 (3), 407-433. Disponible en: http:// www.scielo.org. mx/scielo.php?script $=$ sci_arttext\&pid $=\$ 1665$ 24362006000300005\&lng $=$ en

Power, R. D. \& Dal Martello, M. F. (1990). The Dictation of Italian Numerals. Language and Cognitive Processes, 5 (3), 237-254. Disponible en: http://mcs.open.ac.uk/rp3242/papers/PDM90.pdf

Power, R. D. \& Dal Martello, M. F. (1997). From 834 to Eighty Thirty Four: The Reading of Arabic Numerals by Seven-year-old Children. Mathematical Cognition, 3 (1), 63-85. Disponible en: http://users.mct.open.ac.uk/ rp3242/papers/PDM97.pdf

Scheuer, N.; Sinclair, A.; Merlo de Rivas, S. \& Tièche, C. (2000). Cuando ciento setenta y uno se escribe 10071: niños de 5 a 8 años produciendo numerales. Infancia y Aprendizaje: Journal for the Study of Education and Development, 23 (90), 31-50. DOI: 10.1174/021037000760087955

Seron, X. \& Fayol, M. (1994). Number Transcoding in Children: A Functional Analysis. British Journal of Developmental Psychology, 12 (3), 281-300.

Sullivan, K. S.; Macaruso, P. \& Sokol, S. M. (1996). Remediation of Arabic Numeral Processing in a Case of Developmental Dyscalculia. Neuropsychological Rehabilitation, 6 (1), 27-54.

Tolchinsky, L. (1993). El desarrollo de la escritura. Barcelona: Anthropos.

Tolchinsky, L. \& Karmiloff-Smith, A. (1993). Las restricciones del conocimiento notacional. Infancia y Aprendizaje: Journal for the Study of Education and Development, 62-63, 19-51. Disponible en: http://dialnet.unirioja. es/ejemplar/5452

Zuber, J.; Pixner, S.; Moeller, K. \& Nuerk, H. C. (2009). On the Language Specificity of Basic Number Processing: Transcoding in a Language with Inversion and its Relation to Working Memory Capacity. Journal of Experimental Child Psychology, 102 (1), 60-77. DOI: 10.1016/j. jecp.2008.04.003 


\section{Anexo}

Frecuencias de logro sintáctico de los niños en la escritura de los numerales

\begin{tabular}{|c|c|c|c|c|}
\hline \multirow{3}{*}{ Numeral } & \multicolumn{4}{|c|}{ Frecuencia de logro sintáctico de los niños de cada grado (\%) } \\
\hline & \multicolumn{4}{|c|}{ Grado } \\
\hline & $1^{\circ}$ & $\mathbf{2}^{\circ}$ & $3^{\circ}$ & $4^{\circ}$ \\
\hline 40 & 96,4 & & & \\
\hline 45 & 92,9 & & & \\
\hline 50 & 96,4 & & & \\
\hline 80 & 96,4 & & & \\
\hline 89 & 85,7 & & & \\
\hline 90 & 100,0 & & & \\
\hline 400 & 96,4 & & & \\
\hline 405 & 100,0 & & & \\
\hline 450 & 78,6 & & & \\
\hline 453 & 67,9 & 69,0 & & \\
\hline 500 & 85,7 & 89,7 & & \\
\hline 700 & 96,4 & 96,6 & & \\
\hline 709 & 96,4 & 82,8 & & \\
\hline 790 & 64,3 & 79,3 & & \\
\hline 796 & 67,9 & 79,3 & & \\
\hline 800 & 78,6 & 96,6 & & \\
\hline 2000 & & 93,1 & 100,0 & \\
\hline 2004 & & 69,0 & 96,8 & \\
\hline 2040 & & 48,3 & 96,8 & \\
\hline 2053 & & 51,7 & 90,3 & \\
\hline 2400 & & 82,8 & 93,5 & \\
\hline 2405 & & 65,5 & 93,5 & \\
\hline 2450 & & 55,2 & 100,0 & \\
\hline 2453 & & 62,1 & 96,8 & \\
\hline 3000 & & 79,3 & 96,8 & \\
\hline 8000 & & 72,4 & 93,5 & \\
\hline 8009 & & 48,3 & 90,3 & \\
\hline 8090 & & 44,8 & 90,3 & \\
\hline 8096 & & 44,8 & 83,9 & \\
\hline 8706 & & 69,0 & 93,5 & \\
\hline 8790 & & 55,2 & 100,0 & \\
\hline 8796 & & 72,4 & 96,8 & \\
\hline 8900 & & 65,5 & 96,8 & \\
\hline 9000 & & 86,2 & 100,0 & \\
\hline 70000 & & 79,3 & 100,0 & 100,0 \\
\hline 74528 & & 58,6 & 100,0 & 90,0 \\
\hline 80000 & & 62,1 & 100,0 & 93,3 \\
\hline 80005 & & 51,7 & 90,3 & 93,3 \\
\hline 80045 & & 37,9 & 96,8 & 83,3 \\
\hline 80050 & & 55,2 & 80,6 & 90,0 \\
\hline
\end{tabular}




\begin{tabular}{|c|c|c|c|c|}
\hline \multirow{3}{*}{ Numeral } & \multicolumn{4}{|c|}{ Frecuencia de logro sintáctico de los niños de cada grado (\%) } \\
\hline & \multicolumn{4}{|c|}{ Grado } \\
\hline & $1^{\circ}$ & $2^{\circ}$ & $3^{\circ}$ & $4^{\circ}$ \\
\hline 80400 & & 65,5 & 90,3 & 86,7 \\
\hline 80405 & & 55,2 & 96,8 & 93,3 \\
\hline 80450 & & 58,6 & 96,8 & 96,7 \\
\hline 80453 & & 48,3 & 100,0 & 100,0 \\
\hline 82000 & & 48,3 & 100,0 & 96,7 \\
\hline 82004 & & 44,8 & 100,0 & 90,0 \\
\hline 82040 & & 44,8 & 93,5 & 86,7 \\
\hline 82045 & & 34,5 & 90,3 & 86,7 \\
\hline 82400 & & 69,0 & 96,8 & 100,0 \\
\hline 82405 & & 62,1 & 100,0 & 86,7 \\
\hline 82450 & & 44,8 & 96,8 & 96,7 \\
\hline 82453 & & 72,4 & 100,0 & 93,3 \\
\hline 800000 & & & & 100,0 \\
\hline 874528 & & & & 86,7 \\
\hline 900000 & & & & 96,7 \\
\hline 900005 & & & & 86,7 \\
\hline 900040 & & & & 86,7 \\
\hline 900045 & & & & 83,3 \\
\hline 900400 & & & & 86,7 \\
\hline 900405 & & & & 86,7 \\
\hline 900450 & & & & 93,3 \\
\hline 900453 & & & & 96,7 \\
\hline 902000 & & & & 60,0 \\
\hline 902004 & & & & 66,7 \\
\hline 902040 & & & & 66,7 \\
\hline 902053 & & & & 63,3 \\
\hline 902400 & & & & 66,7 \\
\hline 902405 & & & & 53,3 \\
\hline 902450 & & & & 70,0 \\
\hline 902453 & & & & 40,0 \\
\hline 980000 & & & & 83,3 \\
\hline 980005 & & & & 90,0 \\
\hline 980040 & & & & 73,3 \\
\hline 980045 & & & & 70,0 \\
\hline 980400 & & & & 83,3 \\
\hline 980405 & & & & 83,3 \\
\hline 980450 & & & & 90,0 \\
\hline 980453 & & & & 80,0 \\
\hline 982000 & & & & 83,3 \\
\hline 982004 & & & & 96,7 \\
\hline 982040 & & & & 86,7 \\
\hline 982045 & & & & 90,0 \\
\hline 982400 & & & & 90,0 \\
\hline 982405 & & & & 93,3 \\
\hline 982450 & & & & 93,3 \\
\hline 982453 & & & & 96,7 \\
\hline
\end{tabular}

